\title{
Uso da integração entre Arduino e Matlab no ensino de robótica para engenharias
}

\author{
Use of integration between arduino and matlab in the robotics teaching \\ for engineering
}

1 Atan de Assumção Cardoso atan_cardoso@hotmail.com
1 Adauto Martins de Assis

1 Universidade Federal Fluminense

\section{Resumo}

O mercado de trabalho cada vez mais competitivo tem exigido um alto nível de qualificação dos novos profissionais. É importante que engenheiros estejam capacitados quanto às novas tecnologias utilizadas na indústria. Dentro desse contexto, os autores deste trabalho propuseram a realização de um minicurso de introdução à robótica para alunos de graduação em engenharias. Isso os motivou a realizar um trabalho sobre o uso da integração entre a placa eletrônica arduino e o software Matlab. Ademais, também era necessário explorar a interdisplinaridade, utilizar os recursos pré-existentes na universidade em atividades teóricas e experimentais e buscar ferramentas de programação simplificadas, em razão da baixa carga horária. Os autores optaram por desenvolver atividades através de um braço robótico didático similar aos modelos industriais mais comuns, utilizando a integração entre arduino e Matlab, para tornar os experimentos possíveis. Com base na teoria de análise de posição e outros estudos de robótica, os testes foram realizadas através de toolbox, modelo numérico téorico, modelo 3D e modelo experimental. 0 uso integrado entre arduino e Matlab foi validado por meio da comparação entre resultados teóricos e práticos de cinemática direta e permitiu explorar a interdisciplinaridade, usando os softwares disponíveis na universidade, utilizando apenas uma única linguagem de programação, o que favoreceu o processo de aprendizagem dos alunos.

\section{Palavras-chave}

Robótica. Engenharia. Arduino. Matlab.

\section{Abstract}

The increasingly competitive job market has required a high level of qualification of new professionals. It is important that engineers be trained in the new technologies used by industry. Within this context, the authors of this paper have proposed a minicourse of introduction to robotics for undergraduate engineering students. This motivated them to work on the use of the integration between the Arduino electronic board and the Matlab software. In addition, it was also necessary to explore interdisciplinarity, use the pre-existing resources at the university in theoretical/experimental activities and seek simplified programming tools due to the low workload. The authors chose to develop activities through a didactic robotic arm similar to the most common industrial models, using the integration between arduino and Matlab to make the experiments possible. Based on the theory of position analysis and other robotics studies, tests were performed using a toolbox, theoretical numerical model, 3D model and experimental model. The integrated use between arduino and Matlab was validated by comparing theoretical and practical results of direct kinematics and allowed to explore interdisciplinarity using the software available at the university using only a single programming language, which favored the students learning process.

\section{Como você deve citar?}

CARDOSO, Atan de Assumção; ASSIS, Adauto Martins de. Uso da integração entre Arduino e Matlab no ensino de robótica para engenharias. Cadernos UniFOA, Volta Redonda, n. 42, p. 41-51, abril, 2020.

\section{Keywords}

Robotics. Engineering. Arduino. Matlab. 


\section{INTRODUÇÃO}

Após a revolução industrial, o homem tem buscado alternativas para aprimorar o processo produtivo. Corke (2011) explica que o princípio desse aprimoramento foi o uso de máquinas e ferramentas que puderam facilitar o trabalho humano. No século XX surgiram equipamentos de operação automatizada ou robôs, capazes de atuar com uma menor interferência humana, se comparada com uma simples mecanização.

Desde então, através da evolução tecnológica nas áreas de mecânica, eletrônica, controle, materiais e ciência da computação, houve um aumento na confiabilidade dos robôs e, concomitantemente, foi possível a adição de novas aplicações a esses equipamentos. Robôs de uso industrial tornaram-se essenciais para a realização de atividades automatizadas. Para Sciavicco e Siciliano (2009), entre os principais benefícios da automação através da robótica, estão: redução de custos de produção através da economia de energia; redução do número de pessoas envolvidas no processo; melhor utilização de matéria-prima; aumento da produtividade e do padrão da qualidade; possibilidade de substituir a mão de obra humana em atividades de risco e outras impossíveis de ser controladas manualmente, como montagem de peças em miniatura ou coordenação de movimentos complexos em alta velocidade.

Na opinião de Schwab (2016), a humanidade está vivendo a quarta revolução industrial, conhecida por indústria 4.0, na qual a robótica se destaca entre as grandes inovações tecnológicas. De acordo com o relatório anual da IFR (International Federation of Robotics) sobre o mercado de novos robôs com aplicação industrial em 2018, o Brasil é o país de maior destaque dentro da América do Sul nesse segmento, embora ainda esteja muito atrás de outros países desenvolvidos.

Todos esses equipamentos precisam de mão de obra especializada para operá-los e é fundamental que os universitários tenham, no mínimo, uma formação básica sobre o assunto, sobretudo aqueles que cursam engenharia, pois este é conhecido por ser o profissional responsável por desenvolver soluções técnicas para problemas que possam surgir no ambiente industrial.

No âmbito da aplicação da robótica no Ensino Superior, Piai et al. (2012) e Vargas et al. (2012) conseguiram promover o desenvolvimento de algumas habilidades necessárias a um engenheiro, como trabalho em equipe, raciocínio lógico e pensamento crítico. Ribeiro et al. (2011) realizaram um estudo sobre o uso da robótica como ferramenta de apoio ao ensino de programação e foi observado que os alunos se sentiram motivados e estimulados, pois a abordagem do conteúdo foi realizada de forma diferente da habitual.

Diante dessa necessidade, os autores do presente trabalho propuseram a realização de um minicurso de introdução à robótica a ser disponibilizado aos alunos da Escola de Engenharia Industrial e Metalúrgica de Volta Redonda (EEIMVR), durante a Semana Nacional de Ciência e Tecnologia (SNCT) 2018. Os cursos de engenharia oferecidos na referida instituição são: Metalúrgica, Produção, Agronegócios e Mecânica. Para realização do minicurso, foi disponibilizado o Laboratório de Informática II, que possuía como softwares disponíveis, AutoCAD, SolidWorks, Matlab, pacote MS Office, Arduino IDE, entre outros.

Por tratar-se de um minicurso introdutório para alunos de engenharia, o conteúdo programático teve maior enfoque nos principais conceitos presentes em Fu et al. (1987), Sciavicco e Siciliano (2009) e Niku (2013), como: definição de robô; componentes de um robô; tipos de configurações mais utilizadas na indústria e suas aplicações. Considerando que essa unidade de ensino está situada na região Sul Fluminense, onde há uma gama de empresas com elevado uso de tecnologias no processo de produção, definiu-se que a utilização de um braço robótico didático permitiria aproximar o experimento de aplicações práticas, como pintura e solda a ponto de carrocerias no ramo automotivo. 
Dentro dessa temática, foi observado que, para o braço robótico realizar suas funções, é necessário conhecer a posição do efetuador em relação a um sistema referencial fixo.

O objetivo geral desta pesquisa é utilizar a integração entre a placa eletrônica Arduino e o software Matlab no ensino de robótica para engenharias, com ênfase na análise de posição. Com isso, foram definidos como objetivos específicos: explorar a interdisciplinaridade existente na robótica; realizar análises teóricas e experimentais, utilizando, ao máximo, os recursos disponíveis pré-existentes; buscar ferramentas de programação simplificadas em razão da baixa carga horária do curso.

\section{REVISÃO BIBLIOGRÁFICA}

Em geral, para a análise de posição, foi adotada a metodologia apresentada por Niku (2013) e Fu et al. (1987), composta de um referencial fixo, com três eixos mutuamente ortogonais ( $x, y$ e $z$ ), chamado de $F_{x y z} F_{x y z}$ e outro referencial móvel, chamado de $F_{n o a} F_{n o a}$. A técnica de Denavit-Hartenberg (D-H) é a forma padrão adotada para representar os vários tipos de configurações de robôs ou qualquer robô de malha aberta com quaisquer combinações possíveis de articulações e elos.

O método consiste em atribuir um sistema de referência a cada articulação e, posteriormente, realizar um procedimento que faz a transformação de um sistema para o próximo. Assim, é possível combinar as transformações desde a base até a última articulação, na qual é obtida a matriz de transformação total do robô.

Após a definição dos sistemas de referência, são realizadas quatro operações matriciais para realizar a transformação do sistema $x_{n}-z_{n} x_{n}-z_{n}$ para o sistema $x_{n+1}-z_{n+1} x_{n+1}-z_{n+1}$. 0 procedimento é a mesmo entre dois referenciais quaisquer. 0 produto das matrizes que representam as quatro operações de transformação são representadas em (1) e a transformação entre dois sistemas referenciais consecutivos é chamada $T_{n+1} T_{n+1}$ (2):

$$
{ }^{n} T_{n+1}=\operatorname{Rot}\left(z, \theta_{n+1}\right) \times \operatorname{Trans}\left(0,0, d_{n+1}\right) \times \operatorname{Trans}\left(a_{n+1}, 0,0\right) \times \operatorname{Rot}(x, \alpha
$$

$$
{ }^{n} T_{n+1}=\left[\begin{array}{cccc}
C \theta_{n+1} & -S \theta_{n+1} C \alpha_{n+1} & S \theta_{n+1} S \alpha_{n+1} & a_{n+1} C \theta_{n+1} \\
S \theta_{n+1} & C \theta_{n+1} C \alpha_{n+1} & -C \theta_{n+1} S \alpha_{n+1} & a_{n+1} S \theta_{n+1} \\
0 & S \alpha_{n+1} & C \alpha_{n+1} & d_{n+1} \\
0 & 0 & 0 & 1
\end{array}\right]
$$

em que $n n$ é o número da articulação, $\theta \theta$ é o ângulo de rotação em torno do eixo $z z, \alpha \alpha$ é o ângulo de torção da articulação, aa representa o comprimento de um elo e $d d$ é uma distância sobre o eixo $z z$ que representa o ajuste da articulação. 
A matriz apresentada em (2) pode ser calculada para cada uma das articulações. A transformação total entre a base do robô e o atuador é dada por (3):

$$
{ }^{0} T_{n}={ }^{0} T_{1}{ }^{1} T_{2}{ }^{2} T_{3}{ }^{3} T_{4} \cdots{ }^{n-1} T_{n}
$$

Considerando algumas pesquisas relacionadas à robótica que estão relacionadas a este trabaIho, Allgayer (2011) desenvolveu teoricamente um manipulador robótico cilíndrico com acionamento pneumático, implementando um modelo computacional, utilizando Matlab e Simulink. Foram realizados ensaios variando as cargas dentro do volume de trabalho e, como resultado, o robô alcançou a precisão pretendida dentro do intervalo definido para os diferentes tipos de carregamento.

Nunes (2016) propôs o uso de Redes Neurais Artificiais (RNAs) para realizar o mapeamento da cinemática inversa de manipuladores através de um modelo em Matlab. A referência utilizada por esse autor é um protótipo de cinco graus de liberdade (GDL) desenvolvido em laboratório. Além disso, o autor utilizou uma placa eletrônica Intel Galileo Gen 2 ligada a um notebook. Isso serviu como base para mostrar que é possível criar um código e aplicá-lo a um braço robótico didático através de uma placa eletrônica comercial.

\section{MATERIAIS E MÉTODOS}

Em relação ao cálculo dos resultados teóricos, buscou-se meios de facilitar o ensino de programação. Em razão disso e assim como Allgayer (2011) e Nunes (2016), optou-se por utilizar o software Matlab, devido à disponibilidade de uso e também sua interface amigável e linguagem de programação relativamente simples, sobretudo na criação de gráficos e operações matriciais. Além disso, Corke (2011) fornece um toolbox de robótica para Matlab com diversos recursos, entre eles, o cálculo teórico da cinemática direta e visualização 3D do posicionamento escolhido.

No planejamento da atividade experimental, buscou-se montar um braço robótico didático de configuração versátil e próxima de um modelo industrial. Isso permitiu não somente a verificação e validação dos resultados de posicionamento, mas também examinar outros tipos de falhas que poderiam ocorrer durante o uso e que não foram previstas, tendo em vista que o modelo teórico ideal (material não deformável e ajuste mecânico perfeito) apresenta resultado exato para cinemática direta, desde que os parâmetros de $\mathrm{D}-\mathrm{H}$ tenham sido determinados corretamente.

O modelo desenvolvido possui peças fabricadas em acrílico, fixadas através de parafusos e juntas acionadas por servomotores, braço robótico com cinco graus de liberdade, sendo cinco juntas de rotação e uma garra como efetuador final. Foi criado um modelo 3D utilizando o SolidWorks 2016 x64 edition para permitir aos alunos explorar a geometria e realizar simulações de movimentação, utilizando o recurso controlador de movimentos. Os cinco graus de liberdade são apresentados na figura 1 e as dimensões necessárias para determinar os parâmetros de D-H estão dispostas na figura 2: 
Figura 1 - Graus de liberdade do braço robótico didático.

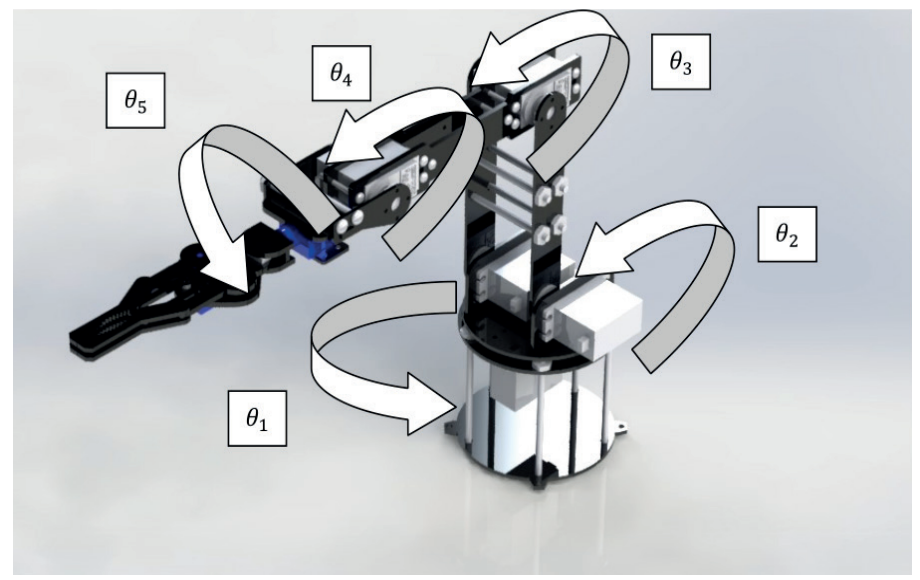

Fonte: autores, 2019.

Figura 2- Principais dimensões para determinar os parâmetros de D-H.

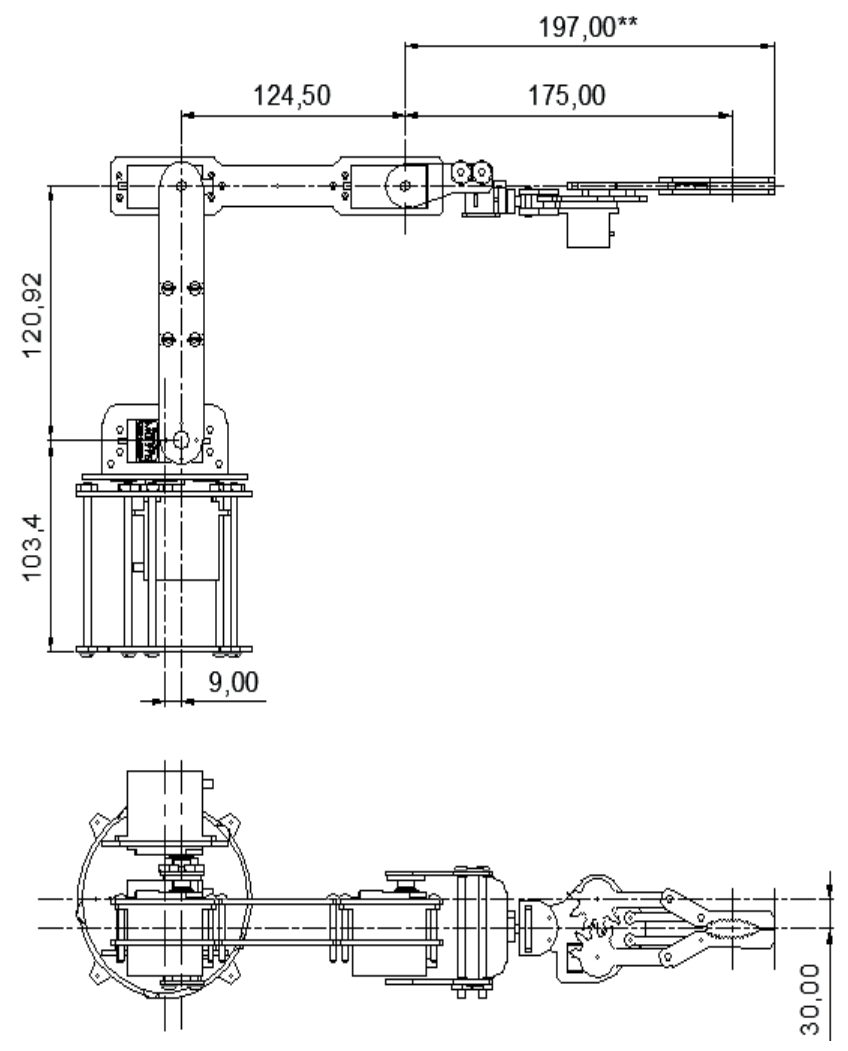

Fonte: autores, 2019.

As principais dimensões das características de construção do robô foram aferidas, utilizando-se um paquímetro analógico do fabricante Starrett de resolução $0,02 \mathrm{~mm}$. As dimensões foram verificadas pelos alunos no modelo físico e no modelo 3D do SolidWorks. Para a posição do efetuador final, foram determinadas duas opções de dimensão. Ao utilizar o centro da garra para transportar um objeto, a cota adotada foi de $175,00 \mathrm{~mm}$ e, ao utilizar a ponta da garra, a cota adotada foi de 197,00 mm. 
Para alcançar os objetivos e tornar os experimentos possíveis, foi desenvolvido um código que explorou a integração entre Matlab e a placa eletrônica Arduino. Isso evitou a necessidade de se trabalhar com duas linguagens de programação diferentes, pois o compilador Arduino IDE fornecido com a placa eletrônica utiliza as linguagens $\mathrm{C} \mathrm{e} \mathrm{C++.} \mathrm{Entre} \mathrm{as} \mathrm{diferentes} \mathrm{placas} \mathrm{Arduino} \mathrm{existentes,} \mathrm{a} \mathrm{Arduino} \mathrm{UNO} \mathrm{foi}$ escolhida em razão de possuir um custo acessível e um número de portas razoável, permitindo diversas aplicações. De acordo com Monk (2017), o fato de a placa Arduino poder ser conectada ao computador através do barramento serial universal (USB) é uma grande vantagem em projetos de eletrônica, pois permite o uso da placa como interface para controlar os mais variados dispositivos.

As ligações do circuito eletrônico foram realizadas com base no circuito apresentado por Mc Roberts (2011) para controle de um servo duplo, porém, nesse caso, o circuito foi adaptado para controle de um total de sete servos, distribuídos na estrutura do braço didático, conforme tabela 1:

Tabela 1 - Aplicação de cada um dos servomotores utilizados no braço robótico didático

\begin{tabular}{cc}
\hline Modelo & Aplicação \\
\hline MG Tower Pro MG 995 & Uma unidade para rotação da base $-\theta_{1}$ \\
\hline JX PDI 300 & Duas unidades em paralelo para movimentação do elo $1 /$ ombro $-\theta_{2}$ \\
\hline MG Tower Pro MG 995 & Uma unidade para movimentação do elo $2 /$ cotovelo $-\theta_{3}$ \\
\hline MG Tower Pro MG 995 & Uma unidade para movimentação do elo 3/punho $-\theta_{4}$ \\
\hline Micro servo 9g SG90 & Uma unidade para rotação da garra $-\theta_{5}$ \\
\hline Micro servo 9g SG90 & Uma unidade para fechamento da garra $-\theta_{6}$ \\
\hline
\end{tabular}

Fonte: autores, 2019.

Após a montagem do circuito eletrônico, o modelo estava apto para análise de posição.

De posse das características de construção do braço robótico didático, foi montada a tabela 2 com os parâmetros de D-H para determinar as equações de cinemática direta:

Tabela 2 - Parâmetros D-H para o braço robótico didático.

\begin{tabular}{ccccc}
\hline Transformações & $\theta$ & $\mathrm{d}$ & $\mathrm{A}$ & $\mathrm{a}$ \\
\hline $0-1$ & $\theta_{1}$ & $\mathrm{~d}_{1}=103,40 \mathrm{~mm}$ & $\mathrm{a}_{1}=9,00 \mathrm{~mm}$ & $\pi / 2$ \\
\hline $1-2$ & $\theta_{2}$ & 0 & $\mathrm{a}_{2}=120,92 \mathrm{~mm}$ & 0 \\
\hline $2-3$ & $\theta_{3}$ & 0 & $\mathrm{a}_{3}=124,50 \mathrm{~mm}$ & 0 \\
\hline $3-4$ & $\left(\theta_{4+} \pi / 2\right)$ & $\mathrm{d}_{4}=30,00 \mathrm{~mm}$ & 0 & $\pi / 2$ \\
\hline $4-5$ & $\theta_{5}$ & $\mathrm{~d}_{5}=175,00 \mathrm{~mm}$ & 0 & 0 \\
\hline
\end{tabular}

Fonte: autores, 2019.

A fim de facilitar a apresentação das matrizes de transformação, foi feita a substituição na descrição das relações trigonométricas de senos e cossenos, onde $\cos \theta_{i}$ e $\operatorname{sen} \theta_{i}$ são representadas, respectivamente, por $\mathrm{C}_{i}$ e $\mathrm{S}_{i}$. Substituindo os valores da tabela na matriz de transformação obtida no 
item 2, obtém-se, então, cinco matrizes, e a transformação entre o referencial fixo e o efetuador final é realizada através das equações (4) e (5):

$$
\begin{gathered}
{ }^{0} T_{5}={ }^{0} T_{1}{ }^{1} T_{2}{ }^{2} T_{3}{ }^{3} T_{4}{ }^{4} T_{5} \\
{ }^{0} T_{5}=\left[\begin{array}{cccc}
s_{1} s_{5}-c_{1} s_{234} c_{5} & s_{1} c_{5}+c_{1} s_{234} s_{5} & s_{1}+c_{1} c_{234} & a_{1} c_{1}+d_{4} s_{1}+a_{2} c_{1} c_{2}+a_{3} c_{1} c_{23}+d_{5} c_{1} c_{234} \\
-c_{1} s_{5}-s_{1} s_{234} c_{5} & -c_{1} c_{5}+s_{1} s_{234} s_{5} & -c_{1}+s_{1} c_{234} & a_{1} s_{1}-d_{4} c_{1}+a_{2} s_{1} c_{2}+a_{3} s_{1} c_{23}+d_{5} s_{1} c_{234} \\
c_{234} c_{5} & -c_{234} s_{5} & s_{234} & d_{1}+a_{2} s_{2}+a_{3} s_{23}+d_{5} s_{234} \\
0 & 0 & 0 & 1
\end{array}\right]
\end{gathered}
$$

onde foram usadas as seguintes simplificações, utilizando as identidades trigonométricas

$$
\begin{aligned}
& \cos \left(\theta_{1}\right)^{*} \cos \left(\theta_{2}\right)-\operatorname{sen}\left(\theta_{1}\right)^{*} \operatorname{sen}\left(\theta_{2}\right)=\cos \left(\theta_{1}+\theta_{2}\right)=\mathrm{C}_{12} \\
& \operatorname{sen}\left(\theta_{1}\right)^{*} \cos \left(\theta_{2}\right)+\cos \left(\theta_{1}\right)^{*} \operatorname{sen}\left(\theta_{2}\right)=\operatorname{sen}\left(\theta_{1}+\theta_{2}\right)=\mathrm{S}_{12}
\end{aligned}
$$

Dessa forma, para cada novo valor da posição angular das juntas, obtém-se a posição final do manipulador. 0 modelo teórico da cinemática direta foi desenvovido separadamente e validado através do toolbox fornecido por Corke (2011), utilizando-se sempre os mesmos parâmetros de entrada em ambos.

Para visualização direta do resultado experimental, o braço robótico foi montado sobre uma placa de cortiça com $40 \mathrm{~mm}$ de espessura e com área plana de $1000 \times 600 \mathrm{~mm}$. Foi impresso um papel milimetrado que foi fixado na superfície da placa, com os eixos também coincidindo com a notação D-H. Desse modo, através da projeção da posição do efetuador no papel milimetrado, eram obtidas as coordenadas dos eixos $x$ e y e, a coordenada em $z$ era obtida através da medição, utilizando-se a distância do efetuador até o plano da placa obtida com régua de acrílico do fabricante Trident de resolução de 1,0mm.

\section{RESULTADOS E DISCUSSÕES}

No código em Matlab, foi criada uma interface com botões em forma de barra deslizante, que permite ao aluno alterar as posições de cada um dos servomotores e também apresentar qual o ângulo atual de cada junta em execução, através da comunicação serial com a placa arduino. 0 botão reiniciar posiciona o braço robótico novamente na posição inicial. A interface é apresentada na figura 3. 
Figura 3 - Interface

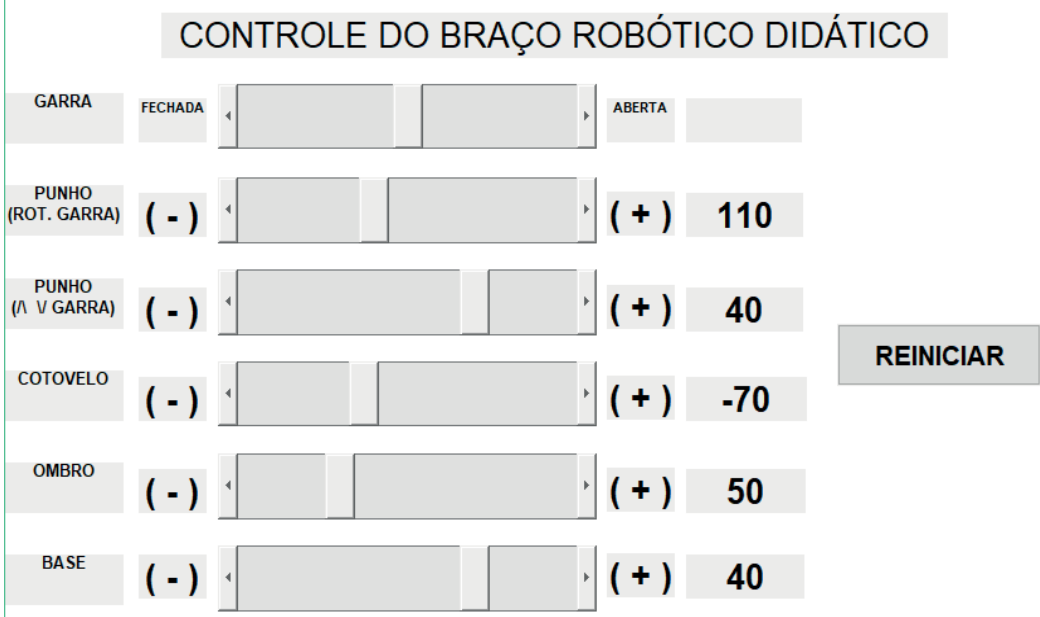

Fonte: autores, 2019.

Nesse ponto, alguns ajustes de posicionamento tiveram que ser inseridos no código, como, por exemplo, o elo 2, ao ser posicionado na posição $90^{\circ}$ na interface, no resultado real, apresentava um ângulo diferente deste. Utilizando-se um goniômetro de aço, faixa de 0 a 180 graus e resolução de $1^{\circ}$ do fabricante Protractor, o elo foi posicionado na posição correta e a diferença de ângulo apresentada anteriormente foi inserida no algoritmo como fator de correção. Além disso, o valor de entrada de alguns servomotores precisou passar por um remapeamento, de forma que os ângulos de rotação passaram a coincir com a notação D-H. Por padrão, os servomotores utilizados trabalham na faixa de 0 a $180^{\circ}$, entretanto, para que a junta 1 trabalhasse com os ângulos coerentes com a notação $D-H$, foi necessário fazer uma alteração para que a faixa de trabalho apresentada na interface fosse de $-90 \mathrm{a}+90^{\circ}$.

O braço robótico didático foi posicionado em diversas posições no toolbox de Corke (2011), no modelo 3D no software SolidWorks, no modelo numérico teórico desenvolvido no Matlab e no modelo experimental. Uma dessas posições escolhida aletatoriamente era formada pelo conjunto de ângulos de rotação das juntas $\theta_{1}=40^{\circ}, \theta_{2}=75^{\circ}, \theta_{3}=-70^{\circ}, \theta_{4}=30^{\circ}$ e $\theta_{5}=10^{\circ}$. Os resultados obtidos por um dos grupos estão dispostos na figura 4 e na tabela tabela 3.

Figura 4 - Uma das Posições utilizadas no experimento - ângulos $\theta_{1}=40^{\circ}, \theta_{2}=75^{\circ}, \theta_{3}=-70^{\circ}, \theta_{4}=30^{\circ} \mathrm{e}$

$$
\theta_{5}=10^{\circ}
$$
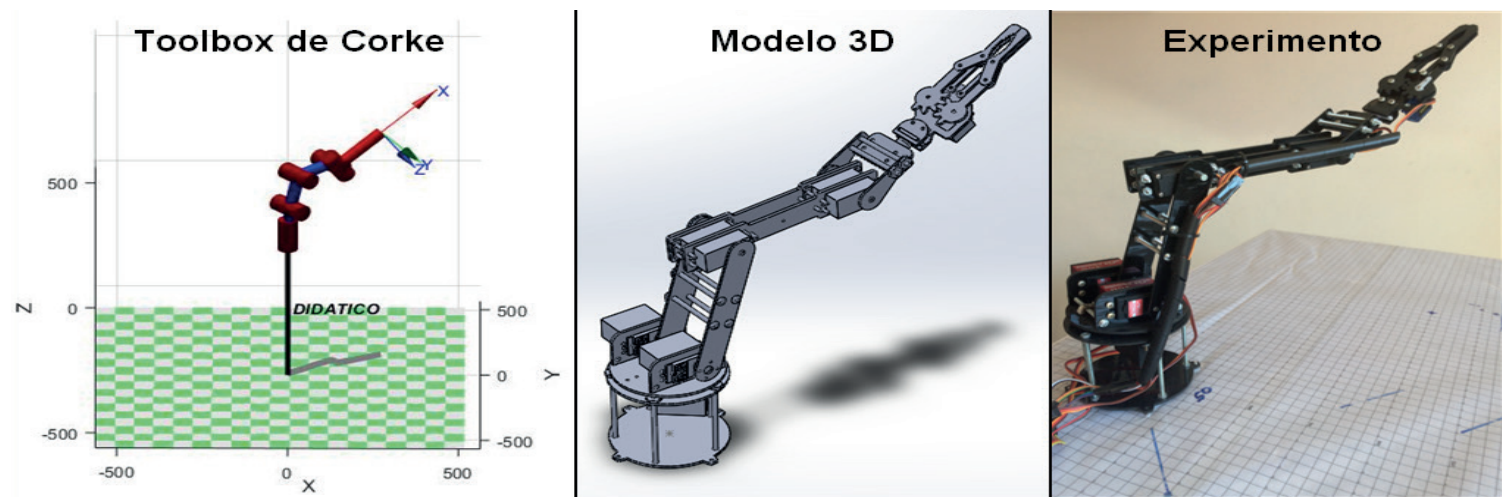

Fonte: autores, 2019. 
Tabela 3 - Comparação de resultados - ângulos $\theta_{1}=40^{\circ}, \theta_{2}=75^{\circ}, \theta_{3}=-70^{\circ}, \theta_{4}=30^{\circ}$ e $\theta_{5}=10^{\circ}$

\begin{tabular}{cccccc}
\hline Coordenada & Toolbox Corke & Modelo teórico & Modelo 3D & Real & Erro \\
\hline$x(\mathrm{~mm})$ & 272,8 & 272,8 & 272,8 & 274,0 & $1,20 \%$ \\
\hline$y(\mathrm{~mm})$ & 150,1 & 150,1 & 150,1 & 151,0 & $0,90 \%$ \\
\hline$z(\mathrm{~mm})$ & 329,9 & 329,9 & 329,9 & 332,0 & $2,10 \%$ \\
\hline
\end{tabular}

Fonte: autores, 2019.

Como era esperado, não houve diferença nos resultados teóricos obtidos no Matlab, seja no toolbox de Corke (2011) ou no modelo teórico numérico desenvolvido através dos cálculos matriciais e no modelo 3D. Em geral, o valor do erro de posicionamento obtido experimentalmente pelos alunos foi satisfatório, com erro menor que $3 \%$ para diversas posições testadas, além da apresentada na figura 4. Esse erro não é significativo, desde que o braço robótico didático seja utilizado em uma aplicação em que o manipulador tenha liberdade para se movimentar sem comprometer o processo, como, por exemplo, movimentação de peças sem obstáculos.

Cabe também ressaltar que foi observado, durante os estudos, que, ao se desligar os servomotores da fonte de alimentação, a posição definida como inicial frequentemente precisava de um novo ajuste, ou seja, sempre antes de realizar novos experimentos era necessário verificar se o posicionamento dos servomotores estava correto. Isso pode ser comparado a uma verificação inicial do robô industrial, após sua instalação na linha de produção e também durante rotina de manutenção.

Assim como no trabalho de Allgayer (2011), foi possível analisar as posições do manipulador através da visualização gráfica no Matlab baseada no modelo teórico, porém apenas no presente trabalho houve verificação experimental para se observar o comportamento em aplicação real do braço robótico. Nunes (2016) realizou cálculos teóricos no Matlab, entretanto utilizou outro software para escrever, compilar e transferir o código para a placa eletrônica, ou seja, foi necessário fazer uso de diferentes linguagens de programação, o que pode gerar alta carga de trabalho, dependendo do tipo de alteração a ser realizada nos códigos. 0 fato deste trabalho fazer uso da integração entre a placa Arduino e o software Matlab aplicada em atividades no ensino de robótica se apresenta como uma valiosa ferramenta didática, permitindo uma análise completa através de resultados numéricos, gráficos animados e resultados experimentais em menor tempo, o que permitiu que o conteúdo básico introdutório fosse abordado em um minicurso utilizando os softwares disponíveis pela universidade, o que atendeu plenamente ao objetivo geral e aos específicos propostos.

Em relação ao conteúdo relacionado à engenharia, os alunos puderam fazer uso da interdisciplinaridade aplicada à robótica através do uso de desenho técnico, cálculos matriciais, programação de computadores, metrologia, eletricidade, eletrônica, dinâmica, física e resistência dos materiais, atendendo perfeitamente o objetivo específico proposto. Assim como nos trabalhos de Ribeiro et al. (2011), Piai et al. (2012) e Vargas et al. (2012), foi possível estimular o pensamento crítico, o trabalho em equipe e o raciocínio lógico.

\section{CONCLUSÃO}

Considerando que o mercado de trabalho está cada vez mais competitivo, apresentando elevado nível de qualificação de novos profissionais, aqueles que estão atualizados sobre as novas tecnologias 
existentes apresentam um diferencial, se comparados aos demais candidatos, pois possuem maior resolutividade. A robótica é uma valiosa ferramenta interdisciplinar capaz de possibilitar ao aluno a capacidade de fazer uma conexão de diversas áreas do conhecimento e suas aplicações práticas.

A principal contribuição deste trabalho foi o uso da integração entre o software Matlab e a placa Arduino como ferramenta de ensino em um curso de Introdução à Robótica para alunos de engenharia, pois permitiu aos participantes visualizarem o comportamento teórico através de resultado numéricos e gráficos e, logo após, o comportamento real de um braço robótico didático em laboratório, tendo sido necessário utilizar somente uma linguagem simplificada de programação com softwares disponíveis na universidade.

O modelo teórico desenvolvido conseguiu apresentar corretamente os resultados de cinemática direta para o braço robótico didático, validado através do toolbox fornecido por Corke (2011). Outros autores como Allgayer (2011) e Nunes (2016) fizeram análises cinemáticas teóricas utilizando Matlab, entretanto não fizeram uso da integração de uma palca eletrônica e Matlab em aplicações experimentais.

Além disso, o braço robótico didático apresentou pequeno erro para o posicionamento previsto para o efetuador final. Esse erro está relacionado ao material de construção, nesse caso, peças de acrílico fixadas através de parafusos e porcas, o que diminuiu a rigidez dos elos e permitiu uma certa deformação, e também os servomotores que sob carga podem apresentar uma certa lentidão para atingir a posição correta. Comparando a um robô industrial, pode ser visto que sua geometria é robusta, de forma a aumentar a rigidez e minimizar o erro por deformação da estrutura. Os resultados também mostram a importância do ajuste mecânico correto e da utilização de um sistema de controle complementar para garantir o posicionamento correto da ferramenta.

Ademais, a análise experimental foi fundamental para verificar problemas de posicionamento do efetuador final que podem acontecer numa situação real e que não estavam previstos. Além disso, implementar a análise cinemática em um braço robótico com Arduino mostrou-se um grande desafio, pois existem diferentes possibilidades de sistema de acionamento, software para realizar a integração e fazer a interface com o usuário etc.

\section{REFERÊNCIAS}

ALLGAYER, R. S. Desenvolvimento de um manipulador robótico cilíndrico acionado pneumaticamente. Dissertação (Mestrado em Engenharia Mecânica). Universidade Federal do Rio Grande do Sul, 2011.

ARDUINO, Página oficial da placa Arduino. Disponível em: https://www.arduino.cc/. Acesso em: 11 set. 2018.

CORKE, P. Robotics, Vision and Control: Fundamental algorithms in Matlab.1 ed. Berlim: Springer-Verlag Berlin Heidelberg, 2011.

FU, K. S., GONZALES R. C., LEE, C. S. G. . Robotics: Control, Sensing, vision and intelligence. 1 ed. Nova Iorque: McGraw-Hill, 1987.

International Federation of Robotics. Executive Summary World Robotics 2018 Industrial Robots . Disponível em: https://ifr.org/downloads/press2018/Executive_Summary_WR_2018_Industrial_Robots. pdf. Acesso em: 15 out. 2018. 
MATLAB. Funções e recursos. Disponível em: https://www.mathworks.com/products/matlab/. Acesso em: 15 out. 2018.

MC ROBERTS, M. Arduino Básico. 1. ed. São Paulo: Novatec, 2011.

MONK, S. Programação com arduino: Começando com sketches. 2. ed. Porto Alegre: Bookman, 2017.

NIKU, S. B. Introdução à robótica: Análise, controle e aplicações. 2. ed. Rio de Janeiro: LTC, 2013.

NUNES, R. F. Mapeamento da cinemática inversa de um manipulador robótico utilizando redes neurais artificiais configuradas em paralelo. Dissertação (Mestrado em Engenharia Elétrica). Universidade Estadual Paulista, 2016.

PIAI, J. C., CERVANTES, S.G.S., FRANÇA, M.B.M, VIEIRA, W.D.C. Uma Experiência com Robótica Educacional no início da formação do Engenheiro Eletricista. Anais do XL Congresso Brasileiro de Engenharia, BelémPA, 2012.

RIBEIRO, P. C., MARTINS, C. B., BERNARDINI, F.C. A Robótica como Ferramenta de Apoio ao Ensino de Disciplinas de Programação em Cursos de Computação e Engenharia. Anais do XXII Simpósio Brasileiro de Informática na Educação, XVII Workshop de Informática na educação, Aracaju-SE, p. 1108 a 1117, 2011.

SCHWAB, K. A Quarta revolução industrial. 1. ed. São Paulo: Edipro, 2016.

SCIAVICCO, L. ,SICILIANO, B. Robotics: Modelling, Planning and Control. 1 ed. Londres: Springer-Verlag London Limited, 2009.

SOLIDWORKS. Dassault Systemes SolidWorks 3D CAD. Disponível em: https://www.solidworks.com/ category/3d-ca. Acesso em: 02 nov. 2018.

VARGAS, M. N., MENEZES, A.G.C, MASSARO, C. M., GONÇALVES, T. M. Utilização da Robótica Educacional como ferramenta lúdica de aprendizagem na Engenharia de Produção: Introdução à produção automatizada. Anais do XL Congresso Brasileiro de Engenharia, Belém-PA, 2012. 